\title{
Students' pre-instructional perspectives of quantum physics
}

\author{
Zac Patterson \\ Department of Teaching and Learning, The Ohio State University, Columbus, OH 43210 \\ Lin Ding \\ Department of Teaching and Learning, The Ohio State University, Columbus, OH 43210
}

Student exposure to modern physics topics (e.g., quantum mechanics, special relativity) is severely limited in k-12 education, but the inclusion of such topics in secondary physics curricula has gained momentum since the turn of the $21^{\text {st }}$ century. Secondary physics curricula in the U.S. predominantly focus on physics content established prior to the $20^{\text {th }}$ century, such as Newtonian mechanics, and rarely address modern topics like quantum physics. Even so, students are still frequently exposed to terms such as quantum physics through a variety of contexts prior to post-secondary education. An analysis of secondary student views of quantum physics may prove valuable to the research community and in the development and implementation of introductory quantum physics curricula. The aim of this study is to analyze secondary student perspectives of the term quantum physics. To do so, a group of secondary physics students were asked to complete a word-association test to establish a baseline of commonalities among student perspectives. The results revealed significant commonalities among student perspectives of quantum physics with the most common association being complexity. The results also revealed that students lack a relevant understanding of quantum physics principles prior to formal instruction and often view the topic as a more sophisticated version of classical physics. 


\section{INTRODUCTION}

A formal introduction to physics would be incomplete without the inclusion of fundamental modern physics topics like quantum physics. Students traditionally do not encounter modern physics topics like quantum physics until late in their secondary education, if at all. In the United States, many of the latest versions of academic content standards for physics are beginning to include some quantum physics content (such as wave-particle duality), but much is still largely left out of secondary physics curricula throughout the country. Apart from the United States, other countries have included a variety of quantum physics topics in physics curricula since the turn of the $21^{\text {st }}$ century, but there is little uniformity among secondary curricula that introduce quantum physics topics [1] and a lack of consensus among researchers on effective instructional practices used to introduce quantum physics [2].

It is easy to dismiss the inclusion of quantum physics at the secondary level, but the privilege of a formal introduction to quantum physics should not solely be available to university physics majors. Quantum physics changes our worldview in a fundamentally new way, and it is becoming increasingly relevant in our daily lives. Quantum physics has played a vital role in current technologies (e.g. medical imaging, nanoscience, and semiconductors) and devices (such as lasers, solar cells, and microchips). It is also the foundation for several emergent technologies including quantum computers, quantum encryption and quantum teleportation.

Research indicates that in order to motivate and attract a larger, more diverse and gender-balanced group of students, physics education needs to focus on knowledge that is relevant to students' lives in the real world [3-5]. Previous studies in Norway $[4,6]$ have shown that quantum physics and relativity are among the topics that engage physics students to a high degree, and students may find these to be more relevant than topics of everyday practical use, such as electricity or friction. Stadermann and Goedhart [7] suggest that quantum physics can have an additional role in the physics curriculum by enhancing students' understanding of nature of science (NOS).

Physics educators may be concerned that quantum physics is too complex and involves mathematical formulism that is beyond the capabilities of most secondary students. These concerns, however, can be put to rest. A stable body of research suggests that quantum physics can be formally introduced in a strictly conceptual manner $[2,8,9,10]$, without the need for advanced mathematical formulism or high levels of complexity. Given this understanding, the time is requisite to expand the inclusion of modern physics topics in secondary schools and to broaden the body of research devoted to the topic.

Inevitably, there are challenges introducing quantum physics to students whose only exposure to formal physics instruction has focused on classical physics topics. After prolonged experience with classical physics topics students often adopt realist perspectives that are in conflict with quantum physics interpretations $[8,11,12]$. In order to comprehend quantum phenomena, an individual must develop a conceptual framework that diverges from classical physics. Since the turn of the $21^{\text {st }}$ century a sizeable body of research has accumulated on quantum physics education. For example, researchers have analyzed students' difficulties [13-15], pedagogical approaches [2,10,16,17], curriculum development [1,18], and epistemological beliefs [19], among other areas of interest. This research has predominately maintained a focus on post-secondary education, with a large amount of work completed at both the undergraduate and graduate level of quantum physics education.

To improve quantum physics instruction and comprehension at the secondary level, a baseline of student preconceptions and perspectives is needed. Once a baseline is established, educators can make research-based decisions that help students establish an appropriate conceptual framework suitable for analysis of quantum phenomena. Establishing this baseline will aid in curriculum development and the development of teaching strategies. To establish a baseline, it would be beneficial to analyze students' perspectives of quantum physics prior to formal instruction.

Understanding the domain specific cognitive structures of student knowledge helps to determine specific features of such knowledge, which can be taken into consideration for instruction [20]. While most students are not formally introduced to quantum physics prior to post-secondary study, they inevitably have been exposed to the term "quantum physics" throughout their lived experiences and have views on the topic. The purpose of this study is to establish some conceptual commonalities that exist among secondary students' pre-instructional perspectives of quantum physics.

\section{METHODOLOGY}

\section{A. Methods}

For this study a continuous, free word-association test and response frequency mapping were used to investigate students' pre-instructional perspectives of quantum physics. A word-association test consists of a word or list of words administered to a participant who responds to the word(s) by means of the first thought coming to his or her mind. A word association test can be of several types depending on its structure. A continuous free word-association test was chosen for this study because it requires that responses not be restricted to predetermined categories, and that there is no limit to the number of responses recorded from each participant. The aim of this study is not to evaluate the relevance and accuracy of participant responses to the stimulus term, but rather to identify the cognitive perspective participants associate with the stimulus term prior to formal instruction. Participant responses to the word-association tests were analyzed and classified into categories. To categorize responses, an iterative process was used to group 
responses sharing similar domain associations. Individual responses that shared no domain associations were dismissed. A response frequency table was then created to illustrate the commonalities and the variety of participant associations to the stimulus term.

\section{B. Sample}

The student sample for this study included 10 secondary students in the $11^{\text {th }}$ and $12^{\text {th }}$ grades attending an urban high school in the Midwestern United States. The students were enrolled in a conceptual-based physics course, had undergone a half-year of physics instruction, and had yet to be formally exposed to quantum physics topics at the time of data collection. Topics covered during the first half of the course included the nature of science, kinematics, forces, electricity and magnetism. The instructor regularly facilitated small group collaborative learning activities with an emphasis on conceptual understanding and inquiry.

\section{Data Collection}

Data collection was completed with a question prompt requesting verbal associations with the stimulus term quantum physics. Each student participant was instructed to verbally announce any words, phrases, ideas or images that came to his or her mind upon hearing the stimulus term. Once students heard the stimulus term, they began to verbally express their associations with the term. The verbal responses were recorded for later categorization. A member check was completed with participants via post-test interviews to increase the validity of response interpretations. Responses were analyzed and classified into categories using an iterative process. A response frequency table was then created to illustrate the commonalities and the variety of participant associations to the stimulus term.

\section{RESULTS AND DISCUSSION}

\section{A. Results}

A total of 44 responses were obtained from the word association tests administered to participants. These 44 responses were categorized based on commonalities and the response frequency for each category was determined (see Table I). A total of 9 categories were created with an average of 4.9 responses per category and an average of 3.8 students providing a response associated with each category. The average number of responses per participant was 4.4 and each participant contributed responses to an average of 3.4 categories.

The data suggests that there are significant commonalities among student associations with quantum physics. This implies that students often obtain similar perspectives of
TABLE I. Categorization of student associations with the stimulus term quantum physics.

\begin{tabular}{|c|c|c|}
\hline Category & $\%$ & Responses \\
\hline $\begin{array}{c}1 . \\
\text { Complex/advanced }\end{array}$ & 22.7 & $\begin{array}{l}\text { Complex equations (x2), } \\
\text { complicated, high } \\
\text { complexity, advanced } \\
\text { study, complex } \\
\text { experiments, large scale } \\
\text { complexity, intricate } \\
\text { science, supreme, } \\
\text { encompassing a lot of } \\
\text { physics }\end{array}$ \\
\hline $\begin{array}{l}2 . \\
\text { Relativity }\end{array}$ & 13.6 & $\begin{array}{l}\text { Space-time }(\mathrm{x} 2) \text {, gravity, } \\
\text { high speed motion, time } \\
\text { dilation, } 4-\mathrm{D} \text { graph }\end{array}$ \\
\hline $\begin{array}{c}3 . \\
\text { Quantum } \\
\text { mechanics }\end{array}$ & 13.6 & $\begin{array}{l}\text { Quantum mechanics } \\
\text { (x2), string theory, light, } \\
\text { uncertainty principle, } \\
\text { superposition }\end{array}$ \\
\hline $\begin{array}{c}4 . \\
\text { Atoms }\end{array}$ & 11.4 & $\begin{array}{c}\text { Atoms (x2), molecular } \\
\text { level, atomic scale, } \\
\text { quarks }\end{array}$ \\
\hline $\begin{array}{l}5 . \\
\text { Popular culture }\end{array}$ & 11.4 & $\begin{array}{l}\text { The Big Bang Theory } \\
\text { (x2), Ant Man (x2), } \\
\text { Quantum Leap }\end{array}$ \\
\hline $\begin{array}{c}6 . \\
\text { Big picture }\end{array}$ & 9.1 & $\begin{array}{c}\text { Big picture, large scale, } \\
\text { how the universe works, } \\
\text { overall }\end{array}$ \\
\hline $\begin{array}{l}7 . \\
\text { Experiments/lab } \\
\text { work }\end{array}$ & 6.8 & $\begin{array}{l}\text { Experimental, high speed } \\
\text { experiments, lab work }\end{array}$ \\
\hline $\begin{array}{c}8 . \\
\text { Proving/developing } \\
\text { theories }\end{array}$ & 6.8 & $\begin{array}{l}\text { Developing and proving } \\
\text { theories, expanding } \\
\text { fundamental principles of } \\
\text { physics, exploring the } \\
\text { unknown }\end{array}$ \\
\hline $\begin{array}{l}9 . \\
\text { Futuristic/sci-fi }\end{array}$ & 4.5 & Futuristic, sci-fi \\
\hline
\end{tabular}




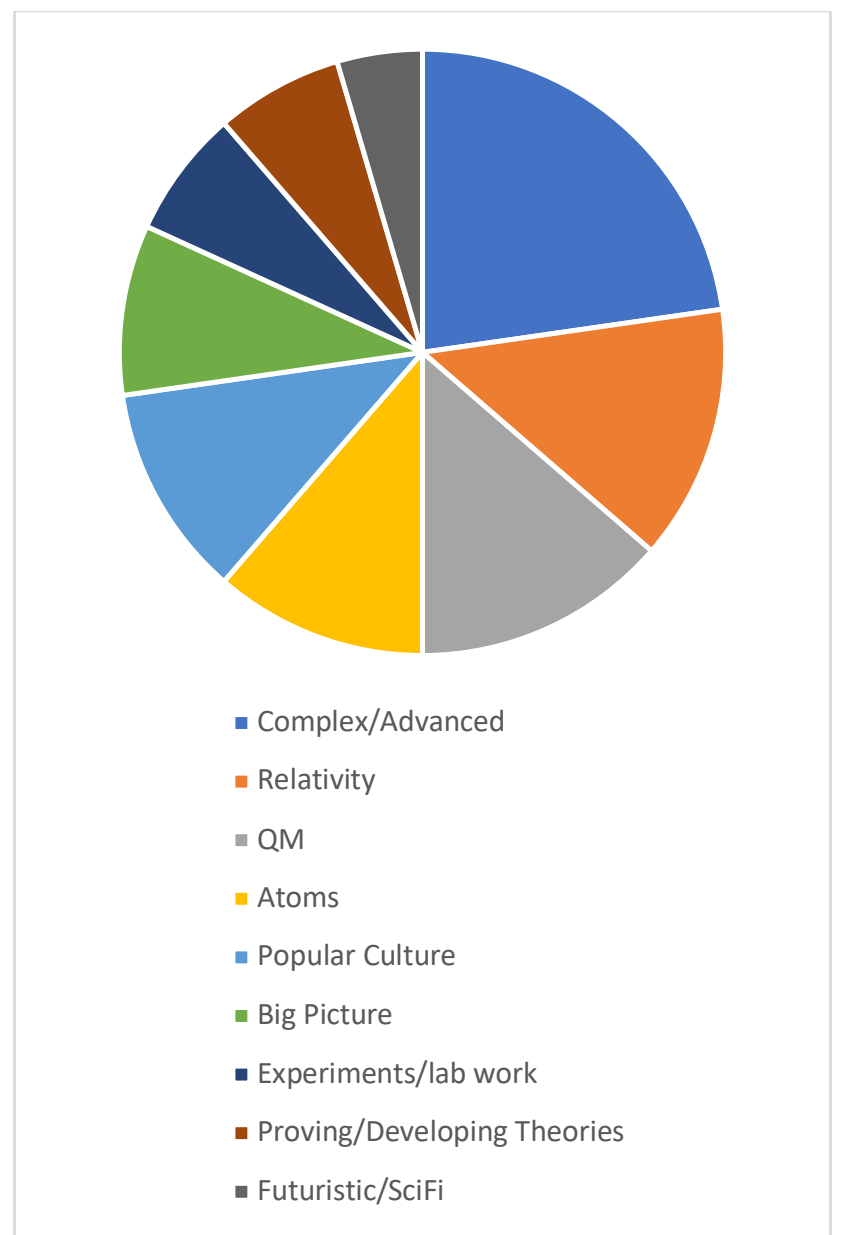

FIG. 1. Response distribution among categories.

quantum physics from their lived experiences prior to formal instruction. These commonalities provide valuable insight into how best to approach introductory quantum physics instruction and improve the efficiency of instruction by building upon existing relevant conceptual structures. Knowledge of these commonalities can also help instructors properly address misconceptions and the conceptual dichotomy that exists between classical physics and quantum physics.

Four of the nine association categories (complex/advanced, relativity, atoms, popular culture) included responses from $50 \%$ of participants, indicating that a significant proportion of students exhibit similar categorical associations to the term quantum physics prior to formal instruction. While member check interviews revealed that students lack an intricate conceptual understanding of the topic, they do have similar cognitive structures pertaining to what they associate with quantum physics. The complex/advanced category had the largest number of associations (10), accounting for $23 \%$ of total responses. Member checks revealed that participants held a common belief that quantum physics is a more sophisticated variation of the classical physics content that had been covered in the course. Participants provided the following responses when asked to elaborate on their associations to the stimulus term that fell in the complex/advanced category:

"What we go over in class is like the fundamentals of physics. But I feel like quantum physics is taking those principles and expanding upon them and making them more complex."

"Like how you had us do the straw rockets, that's something that is basic. . like (quantum physics) would have us do that and then explain how Nasa would use rockets to go to space."

"What we do in our class is a smaller scale than quantum physics."

"Intricate science. More in depth."

"Like the equations we do in physics now. . but they go on."

\section{B. Discussion}

The belief that quantum physics is a complex version of classical physics can implicate the teaching of introductory quantum physics. Introductory quantum physics need not be complex, but the concepts associated with the topic do clash with canonical physics views. A realist perspective may be sufficient to conceptualize classical physics topics, but quantum phenomena require an alternative conceptual framework for comprehension. Research [11] suggests that the realist perspectives students often obtain from studying classical physics are often inappropriately associated with quantum phenomena. Instructors must account for this in their approach to introducing quantum physics topics and make the differences explicitly clear. To help alleviate the challenges associated with a formal introduction to quantum physics it may be beneficial to introduce quantum physics topics earlier in students' academic careers, before classical frameworks are solidified, making an appropriate conceptual framework of the quantum realm difficult to obtain. A handful of countries, such as Norway, Belgium and the Netherlands, have successfully included quantum physics topics in upper level secondary physics curricula for more than two decades, with a focus on conceptual understanding [21]. It may be time for more curriculum developers to seriously consider introducing quantum physics earlier in students' academic careers and with more depth.

The common belief that quantum physics is solely studied by advanced scientists inevitably arises from associations with quantum physics in popular culture along with a lack of formal introduction to the topic earlier in students' academic careers. In order to successfully implement quantum physics into secondary physics classrooms, instructors need to illustrate that the concepts are obtainable for students. Building upon prior knowledge such as the quantization of elementary particles or electron energy levels may help ease the transition to quantum study. Taking advantage of the growing body of open source quantum physics simulations and software may also help introduce the topic in a more comprehensible and less intimidating manner. 
Although some commonalities were found among associations within this study, a lack of relevance to quantum physics principles was evident among student perspectives. This is not surprising given the lack of formal instruction on the topic, but it does indicate that participants were unaware of some of the key concepts associated with quantum physics. For instance, there seems to be a lack of association with quantization. None of the 10 student participants made any associations with the literal meaning of the word quantum. Given the importance of the natural laws of quantization that govern the quantum realm this seems to be an area worth further exploration. Students inevitably are exposed to the idea of quantization prior to upper secondary school study, with introductions to electron energy levels and the standardized mass units associated with elementary particles, but they do not seem to associate this meaning with the term quantum. With quantization being a fundamental component of quantum physics this deserves to be an area of focus for introductory quantum physics courses and could perhaps be used as an initial instructional foundation to build upon.

The fact that students exhibited a strong association with relativity may indicate that students confuse it with quantum physics and lump the two into one. This is not surprising given the lack of instruction on modern physics in secondary curricula. It is important for instructors to distinguish the differences among modern physics topics such as quantum physics and relativity, and there may be opportunity to use quantum physics as a segue to other modern physics topics, like relativity.

\section{Conclusion}

It is not surprising that secondary students do not hold a strong conceptual understanding of quantum physics prior to formal instruction on the topic. Contemporary physics topics are largely left out of secondary physics curricula and students' exposure to these modern topics is almost entirely absent in their formal education prior to physics at the secondary level. The result that students largely associated quantum physics with a complex, or more advanced version of classical physics is the key finding of this study. There are several implications associated with this finding with regards to instruction. First, instructors must find a way to facilitate the conceptual change necessary to interpret quantum phenomena. Students must establish a conceptual framework that diverges from the familiar deterministic nature of classical mechanics. Secondly, the preconception that quantum physics is complex and only comprehensible by trained physicists needs addressed. As the previously cited research $[2,8,9,10]$ illustrates, quantum physics can successfully be taught to secondary students with an emphasis on conceptual understanding and without the inclusion of complex mathematics. To achieve this, students need to believe they are capable of understanding the content. Lastly, pre- and in-service teacher training may need to address the necessary divergence from a classical mechanics framework when addressing quantum topics. This conceptual change is unique to contemporary physics topics and likely to be overlooked by many secondary physics instructors.

How best to incorporate quantum physics into secondary curricula is a question that further research will need to address. A body of research is accumulating on the topic, but it is of relatively novel interest to physics education researchers. The inclusion of contemporary physics topics in secondary physics curricula has increased in many areas of the world since the turn of the $21^{\text {st }}$ century and this trend is likely to continue as quantum technologies and applications become increasingly relevant in modern society. The instructional techniques, learning materials, and course progressions that work best for secondary students and instructors are actively under study and void of consensus.

While this research has the potential to be a focal point for further inquiry, it must be recognized that the results are preliminary, and the sample size was relatively small for a study of this nature $(n=10)$. Nonetheless, the study revealed valuable insight regarding the research focus.

\section{ACKNOWLEDGEMENTS}

We would like to thank all of the students who participated in this study. 
[1] K. Krijtenburg-Lewerissa, H.J. Pol, A. Brinkman, and W.R. van Joolingen, Key topics for quantum mechanics at secondary schools: a Delphi study into expert opinions, Int. J. Sci. Educ. 41, 3 (2019).

[2] K. Krijtenburg-Lewerissa, H.J. Pol, A. Brinkman, and W.R. van Joolingen, Insights into teaching quantum mechanics in secondary and lower undergraduate education, Phys. Rev. - PER. 13, 1 (2017)

[3] K. Stokking, Predicting the choice of physics in secondary education, Int. J. Sci. Educ. 22, 12 (2000)

[4] C. Angell, Ø. Guttersrud, E.K. Henriksen, and A. Isnes, Physics: Frightful, but fun. Pupils' and teachers' views of physics and physics teaching, Sci. Educ. 88, 5 (2004)

[5] M.V. Bøe and E.K. Henriksen, Love it or leave it: Norwegian students motivations and expectations for postcompulsory physics, Sci. Educ. 97, 4 (2013)

[6] R. Renstrøm, Ph.D. thesis, University of Oslo, 2011.

[7] H.K.E. Stadermann and M.J. Goedhart, Secondary school students' views of nature of science in quantum physics, Int. J. Sci. Educ. (2020)

[8] R. Muller and H. Wiesner, Teaching quantum mechanics on an introductory level, Amer. J. Phys. 70, 3 (2002)

[9] G. Kalkanis, P. Hadzidaki, and D. Stavrou, An instructional model for a radical conceptual change towards quantum mechanics concepts, Sci. Educ. 87, 2 (2003)

[10]E.K. Henriksen, B. Bungum, C. Angell, C.W. Tellefsen, T. Fragat, and M.V. Bøe, Relativity, quantum physics and philosophy in the upper secondary curriculum: challenges, opportunities and proposed approaches, Phys. Educ. 49, 6 (2014)

[11]C. Baily and N.D. Finkelstein, Teaching and understanding of quantum interpretations in modern physics courses, Phys. Rev. - PER. 6, 1 (2010).

[12] C. Baily and N.D. Finkelstein, Teaching quantum interpretations: revisiting the goals and practices of introductory quantum physics courses, Phys. Rev. PER. 11, 2 (2015)

[13] I.D. Johnston, K. Crawford, and P.R. Fletcher, Student difficulties in learning quantum mechanics, Int. J. Sci. Educ. 20, 4 (1998)

[14] K. Mannila, I.T. Koponen, and J.A. Niskanen, Building a picture of students' conceptions of wave- and particle-like properties of quantum entities. Eur. J. Phys. 23, 45 (2001)

[15] S. Wuttiprom, M.D. Sharma, I.D. Johnston, R. Chitaree, and C. Soankwan, Development and use of a conceptual survey in introductory quantum physics, Int. J. Sci. Educ. 31, 5 (2009)
[16]B. Bungum, E.K. Henriksen, C. Angell, C.W. Tellefsen, and M.V. Bøe, ReleQuant - improving teaching and learning in quantum physics through educational design research, Nordic Stud. Sci. Educ. 11, 2 (2015)

[17] W. Dür and S. Heusler, The qubit as key to quantum physics part II: Physical realizations and applications, Phys. Teach., 54, 3 (2016)

[18] G. Pospiech, Teaching quantum theory - between the photoelectric effect and quantum information, GIREP 2008 Conference Proceedings

[19]B.W. Dreyfus, J.R. Hoehn, A. Elby, N.D. Finkelstein, and A. Gupta, Splits in students' beliefs about learning classical and quantum physics, Int. J. STEM Educ. 6, 1 (2019)

[20]A. Derman and I. Eilks, Using a word association test for the assessment of high school students' cognitive structures on dissolution, Chem. Educ. Res. \& Prac. 17, 4 (2016)

[21] H.K.E. Stadermann, E. van den Berg, and M.J. Goedhart, Analysis of secondary school quantum physics curricula of 15 different countries: different perspectives on a challenging topic. Phys. Rev. PER. 15, 1 (2019) 